Article

\title{
Design of Low-Cost Synchronous Machine to Prevent Demagnetization
}

\author{
Claudio Bianchini ${ }^{1}$, Ambra Torreggiani ${ }^{1}\left(\mathbb{D}\right.$, Matteo Davoli $^{2}$ and Alberto Bellini ${ }^{3, *(1)}$ \\ 1 Department of Engineering “Enzo Ferrari” (DIEF), University of Modena and Reggio Emilia, \\ 41125 Modena (MO), Italy; claudio.bianchini@unimore.it (C.B.); ambra.torreggiani@unimore.it (A.T.) \\ 2 Raw Power Srl, Via Sicilia 31, 42122 Reggio Emilia (RE), Italy; matteo.davoli@rawpowergroup.it \\ 3 Department of Electrical, Electronic, and Information Engineering “Guglielmo Marconi” (DEI), University of \\ Bologna, 47521 Cesena (FC), Italy \\ * Correspondence: a.bellini@unibo.it; Tel.: +39-0547-339229
}

Received: 15 June 2020; Accepted: 8 July 2020; Published: 10 July 2020

check for updates

\begin{abstract}
The request for high efficiency motor paves the way for the replacement of induction motors with permanent magnet synchronous motors. Although the efficiency is increased, for medium and high power, the current ripple causes significant additional losses in the magnet and lamination; and, high temperature can lead to demagnetization. In this paper, a new rotor topology is proposed and compared to a traditional surface permanent magnet rotor to reduce the magnet losses and protect them from demagnetization. A reference surface permanent magnet machine is compared with the proposed one in terms of performance and magnet losses. Both analytical and experimental analysis are carried out and discussed.
\end{abstract}

Keywords: permanent-magnet synchronous motors 1; efficiency class 2; iron losses 3; magnet eddy currents 4

\section{Introduction}

The International Electrotechnical Commission (IEC) technical committee has estimated that electric motors cover the $50 \%$ of the global electric consumption, which is split in industrial electric motors (30\%) and others (20\%). As a consequence, the urge of reducing global greenhouse emissions paves the way to the demand of more efficiency in electric motor design.

In the last twenty years, the improvement of the standardized efficiency class of induction motors (IM) [1] from IE0 class to IE3 class was accomplished by different techniques:

- $\quad$ increasing the motor total stack length for the same output power;

- changing the design of rotor and stator lamination;

- employing low losses silicon iron steel; and,

- $\quad$ substituting the aluminum of the squirrel cage bars with copper bars.

Nevertheless, the use of the most advanced materials together with variable speed drives lead to new scenarios for high efficiency permanent-magnet (PM) synchronous electric motors.

Permanent-magnets synchronous electric motors are intrinsically more efficient than induction motors because of the absence of rotor electric circuit and, consequently, of Joule losses. Moreover, permanent-magnets synchronous electric motors have higher power factor, since they do not require a magnetization component in the current.

In this framework, some electric motor manufacturers have decided to move from induction motors to permanent-magnets motors, even for low-cost applications. Authors of [2] show several different low-cost configurations while using a traditional induction motor mechanical frame and stator 
lamination for permanent-magnets synchronous motor. The proposed procedure aims at realising a "low-cost" machine, thanks to two major choices. At first, the machine is designed re-using stator and frame from a reference induction machine, which has a very standardized manufacturing process. Second, magnet segmentation is avoided, because it would result in high manufacturing cost for medium-large machines.

Although permanent-magnets synchronous motors potentially present a better efficiency than induction motors, their intrinsic lower phase inductance results in a higher current ripple at fixed Pulse Width Modulation (PWM) switching frequency. The current ripple causes additional iron losses in the laminations and large eddy current losses inside permanent-magnets [3-7], even if permanent-magnets conductivity is lower than that of winding materials (i.e., aluminum and copper). Eddy current losses are quite critical in permanent-magnets synchronous motors with distributed winding topology [3], such as those made starting from induction motors mechanical frame. These addition losses could affect motor global efficiency and even lead to permanent-magnets demagnetization due to a very high working temperature.

This paper shows an extensive investigation of permanent-magnet demagnetization issue in a low-cost surface permanent-magnet (SPM) synchronous motor with distributed winding at different PWM frequencies and current ripple amplitudes. A different rotor design configuration is proposed in order to reduce permanent-magnets eddy current losses, thus protecting them from demagnetization. The proposed rotor is compared to a reference surface permanent-magnet motor, via finite element analysis (FEA) and experimental tests.

This paper is organized, as follows: Section 2 shows a brief analysis of design techniques to reduce permanent-magnets eddy current losses, Section 3 shows the finite-element analysis of reference and proposed motors, Section 4 shows experimental results, and Section 5 draws some conclusions.

\section{Design Techniques to Reduce Permanent-Magnets Eddy Currents}

Current ripple losses could significantly affect the global motor efficiency and lead to PMs demagnetization because of the increase of magnet working temperature. Moreover, the current ripple increases with the machine size for two main reasons: reduction of phase resistance and inductance, and effect of reduction of PWM switching frequency to contain switching losses as rated current increases.

For these reasons, some specific design solutions shall be adopted as the machine size increase. Several state-of-the-art solutions exist to limit the eddy currents in magnets: PMs segmentation along axial or circumferential direction. However, the segmentation suffers from eddy current reaction field, which superimposes itself to stator magnetic field, as shown in [5], thus reducing the effectiveness of segmentation. This phenomenon is related to the inductive and resistive behavior of eddy current paths that grows as the eddy current frequency increases.

The skin effect is augmented by eddy current reaction field, thus reducing the effectiveness of PMs segmentation. In [6-8], it is shown that PMs segmentation is only feasible when skin effect is negligible. In [8], it is shown that a small number of PMs segmentation increases eddy current losses, instead of diminishing it as expected, when the skin effect is significant. This phenomenon is defined by the authors as "anomaly of segmentation". The anomaly of segmentation is also reported in papers [3,6], which analyze the effects of harmonics order and of rotor type (e.g., surface and internal PMs rotor) on PMs loss reduction. Figure 1 shows profiles of eddy current losses as a function of number of PMs segmentation: the orange curve is typical of low-frequency harmonics (e.g., stator slot harmonics), while the blue curve is typical of high-frequency harmonics (e.g., PWM switching frequency). The anomaly of segmentation is represented by the blue curve, whose peak value occurs with a fixed PMs segmentation. 


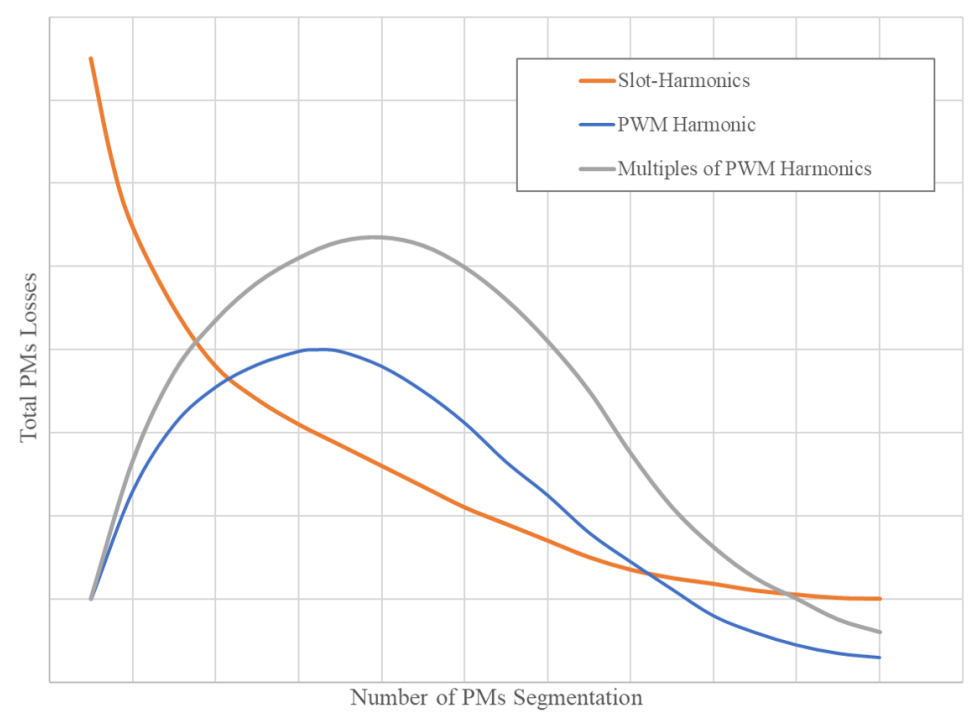

Figure 1. Permanent-magnets eddy current losses profiles as a function of magnet segmentation. The orange curve represents the profile of low-frequency harmonics and blue and grey curves represent the profile of high-frequency harmonics [5].

According to the current state of knowledge, the maximum of eddy current losses occurs when PMs segmentation is nearly twice the skin depth $\delta(1)$, where $\rho$ is the PM resistivity, $\mu$ is the magnetic permeability, and $f_{e l}$ is the electric frequency.

$$
\delta=\sqrt{\frac{\rho}{\pi f_{e l} \mu}}
$$

Hence, the magnet segmentation improves eddy currents losses only if it is smaller than twice the skin depth, [6]. Moreover, as the high-frequency harmonic order increases the peak of the blue curve shifts to the right, thus a finer PM segmentation could exacerbate PM eddy current losses, while a reduction would be expected. This behavior is represent by the grey curve of Figure 1 .

Another design solution to reduce PM eddy currents is interior permanent-magnet (IPM) rotor topology. In fact, the iron region around the magnets of IPM rotor acts as a harmonic filter protecting magnets from additional eddy currents. Papers [7,9] show that IPM rotor has a lower PM losses component than inset and SPM rotors because of the iron regions that protects PM from slot and carrier harmonics. This phenomenon is enhanced for IPM synchronous machines with concentrated winding, since they feature a high space harmonic content in magnetomotive force (MMF).

Both magnet segmentation and rotor design can be effectively used to reduce eddy currents. The paper is investigating the latter only, because it is focused on low cost solutions. Magnet segmentation is quite expensive for medium-large machines.

\section{Reference and Proposed Electric Motors}

The performance the reference low-cost surface permanent-magnet motor is analyzed and compared with that of the proposed IPM motor. Both motors have 36-6 slot-pole combination, the same stator lamination and mechanical frame, and a distributed winding topology. Table 1 summarizes the main characteristics of the machines. The comparison is based on a number of fixed parameters: same stator lamination geometry, same winding configuration, same slot filling factor, and same output power. 
Table 1. Characteristics of reference surface permanent-magnet (SPM) and proposed interior permanent-magnet (IPM) motors.

\begin{tabular}{cccc}
\hline Parameters & Reference SPM & Proposed IPM & Unit of Measurement \\
\hline Stator Slots & 36 & 36 & - \\
Pole Pairs & 3 & 3 & - \\
Slots per Phase per Pole (SPP) & 2 & 2 & - \\
Stator Outer Diameter & 210 & 210 & $(\mathrm{~mm})$ \\
Lamination Stack Length & 100 & 100 & $(\mathrm{~mm})$ \\
PMs Total Volume & $2.646 \times 10^{-4}$ & $2.638 \times 10^{-4}$ & $\left(\mathrm{~m}^{3}\right)$ \\
Phase to phase resistance & 0.31 & 0.31 & $(\Omega)$ \\
Direct axis inductance & 2.3 & 4.2 & $(\mathrm{mH})$ \\
Quadrature axis inductance & 2.3 & 4.7 & $(\mathrm{mH})$ \\
\hline
\end{tabular}

\subsection{Reference Surface Permanent-Magnet Synchronous Motor}

The reference machine has a standard $132 \mathrm{~mm}$ shaft height mechanical frame with wings and fan. The magnet type is $\mathrm{NdFeB} 32 \mathrm{SH}$. Figure 2a shows reference machine geometry.

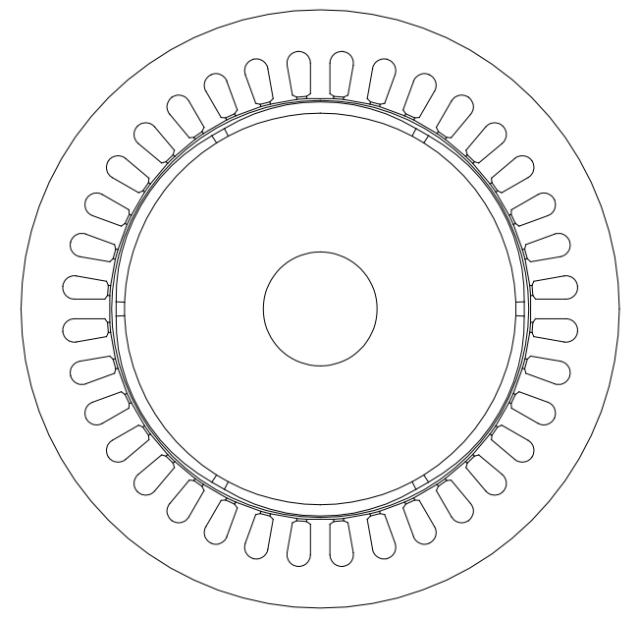

(a)

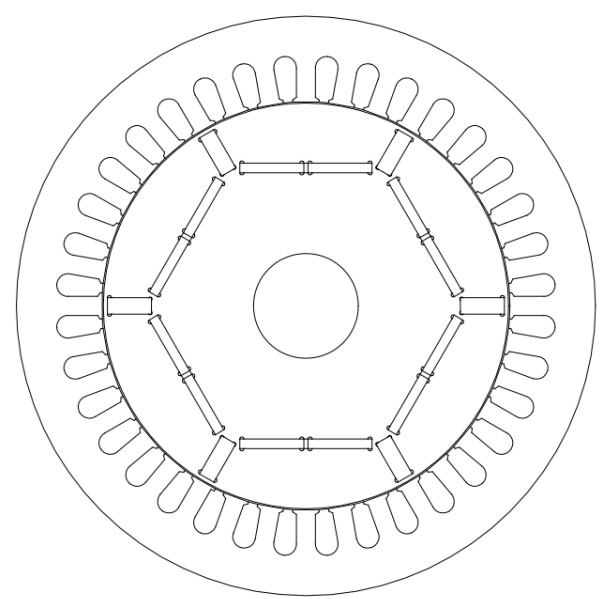

(b)

Figure 2. 2D geometry of: (a) reference surface permanent-magnet synchronous machine; (b) proposed Interior Permanent-Magnet Synchronous Machine.

The magnet flux distribution at no load is shown in Figure 3a. Although the stator lamination are taken from a IM, its saturation level is adequate and consistent with the silicon steel material M570-50A used in the prototype, in order to reduce overall motor costs.

As demonstrated by the experimental results, when the SPM machine is fed with an industrial drive with a PWM frequency equal to $4 \mathrm{kHz}$, the induced stator current ripple drives magnet temperature over $150^{\circ} \mathrm{C}$. Figure 4 shows how magnets reached working temperature around $177^{\circ} \mathrm{C}$. As a consequence, PMs working point falls below the knee of demagnetization curve of B-H curve and PMs are irremediably demagnetized. 


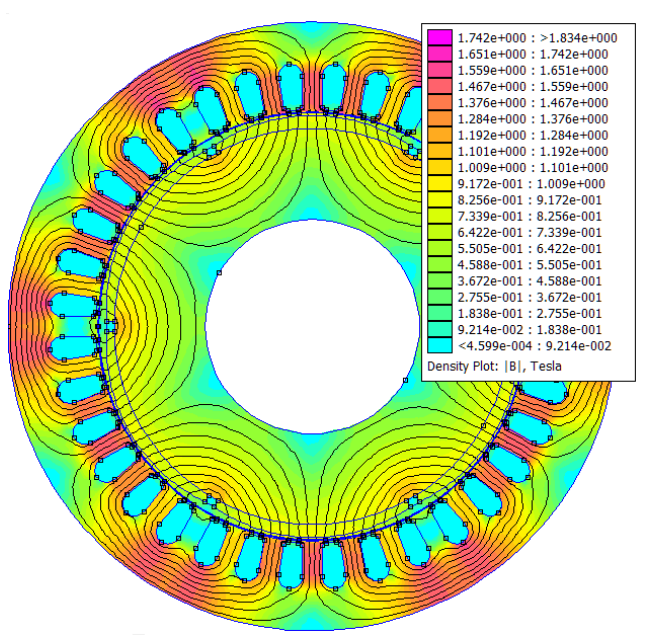

(a)

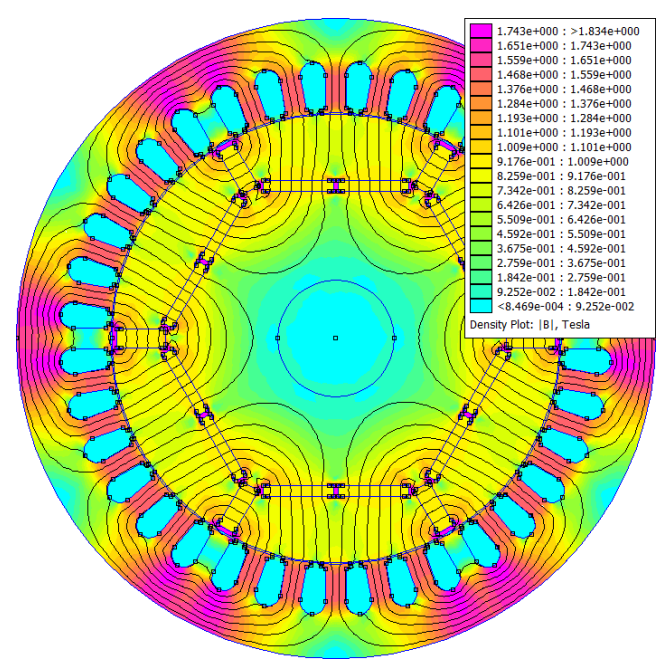

(b)

Figure 3. Flux density distribution from $0.84 \mathrm{mT}$ to $1.83 \mathrm{~T}$ at no load of: (a) reference surface permanent-magnet synchronous machine; (b) Proposed Interior Permanent-Magnet Synchronous Machine.

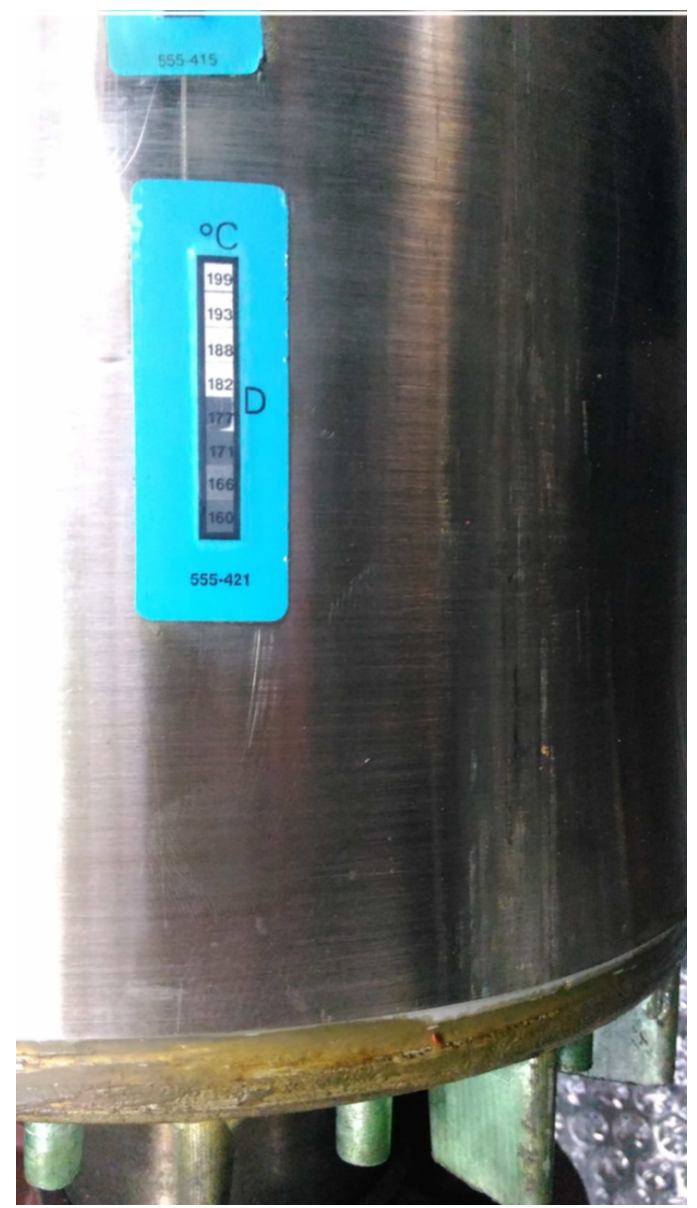

Figure 4. SPM motor rotor thermal label after test at $4 \mathrm{kHz}$.

In order to overcome this issue this paper proposes a different rotor configuration where PMs are deep into the rotor, so that they are protected from additional eddy current losses. 


\subsection{Proposed Interior Permanent-Magnet Synchronous Motor}

The three major benefits of the IPM rotor are:

- $\quad$ increased synchronous inductance;

- $\quad$ increased PMs protection thanks to iron region above them [9]; and,

- no need of the retaining sleeve, that can induce additional eddy currents, since generally is manufactured with staining steel or copper $[5,10]$.

Several geometries can be used for magnet location in a IPM configuration, as [7,11]. Here, the spoke rotor configuration was used as a starting point for the optimizazion process. Prior art reports other rotor designs (still within IPM machine type), however, we have chosen a rotor configuration that can achieve similar performances to a SPM, without increasing magnet size and cost.

The proposed IPM rotor has been designed to reduce the leakage flux of PMs and increase its mechanical stress resistance during spinning. Figure $5 b$ shows the magnetic flux paths of PMs: the height of radial PMs has been shortened and transverse PMs have been placed between them in order to reduce the leakage magnetic flux.

Figure $2 b$ shows the proposed IPM geometry and Figure $3 b$ no load magnetic flux distribution. The basic design for the IPM rotor is with radial PMs direction and it is usually referred to as "spoke rotor", see Figure 5a. The paths of the magnetic flux and the principle of PMs orientation are also shown in Figure 5a. The spoke rotor type intrinsically has a certain amount of leakage magnetic flux that does not produce electro-mechanical torque (red solid arrows of Figure 5a). Moreover, the thin lamination ring near rotor shaft is mechanically weakened in order to increase the reluctance of the leakage magnetic flux paths. Figure 6 shows the mechanical stress on the spoke rotor caused by centrifugal forces $\overrightarrow{F_{C}}$ during rotor spinning.

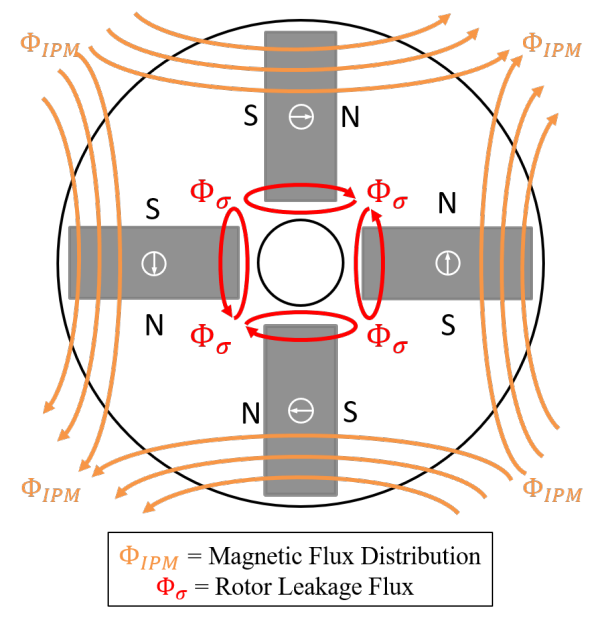

(a)

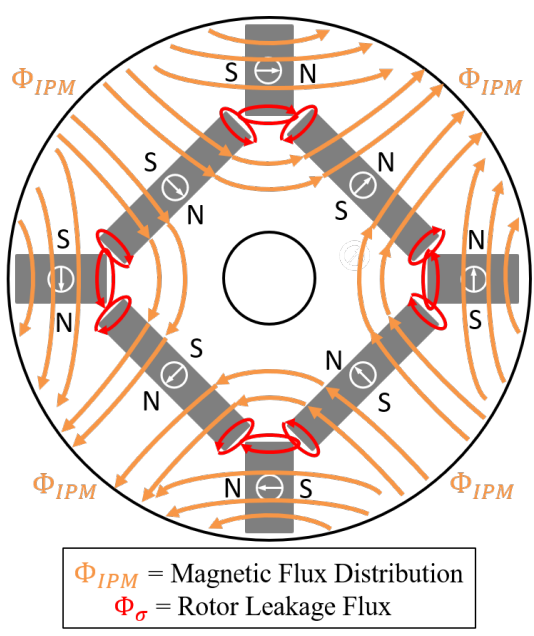

(b)

Figure 5. Representation of magnetic flux paths of: (a) IPM rotor with radial permanent-magnet; (b) proposed IPM rotor. 


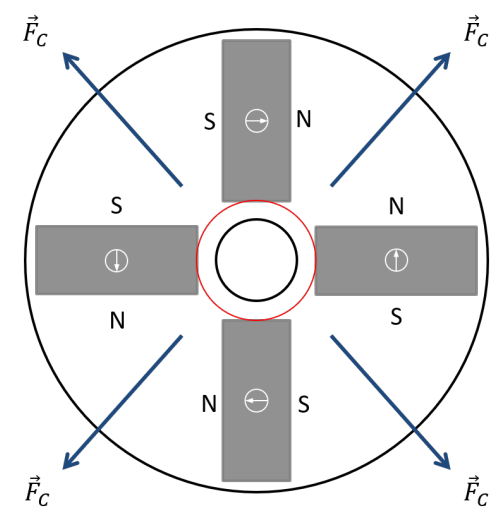

Figure 6. centrifugal force $\overrightarrow{A_{C}}$ acting on spoke rotor.

\section{Finite-Element Analysis and Performance Comparison}

\subsection{Magneto-Static Analysis of Reference and Proposed Machines by FEA}

The reference SPM and the proposed IPM machines were designed and compared via FEA fixing root mean square (RMS) current density at $6.7 \mathrm{~A} / \mathrm{mm}^{2}$, with a winding factor of 0.35 and a mechanical speed of $3000 \mathrm{rpm}$, that is the reference speed for many applications with IM fed at $50 \mathrm{~Hz}$. The machines were simulated with a pure sinusoidal stator current and with a current angle that corresponded to a zero component on d-axis (field axis of the permanent magnet on the rotor reference frame).

Simulations were carried out including the current ripple generated by the power converter, in order to assess the effects of switching operation on motor losses, Figure 7. The ripple on currents in in d-q reference frame appears in quadrature because of the phase shift chosen on the stator three-phase current system. A current ripple of identical quadrature is superimposed to the three phase currents with different angle according to the reference phase angle. The angle of phase current vector of the proposed IPM was $90^{\circ}$, because the saliency ratio $\xi$ is close to unity, thus the only component of current is on q axis, as for SPM.The ripple of stator current was computed by numerical simulation with a lumped parameter model of the Voltage Source Inverter (VSI) at different PWM switching frequencies.

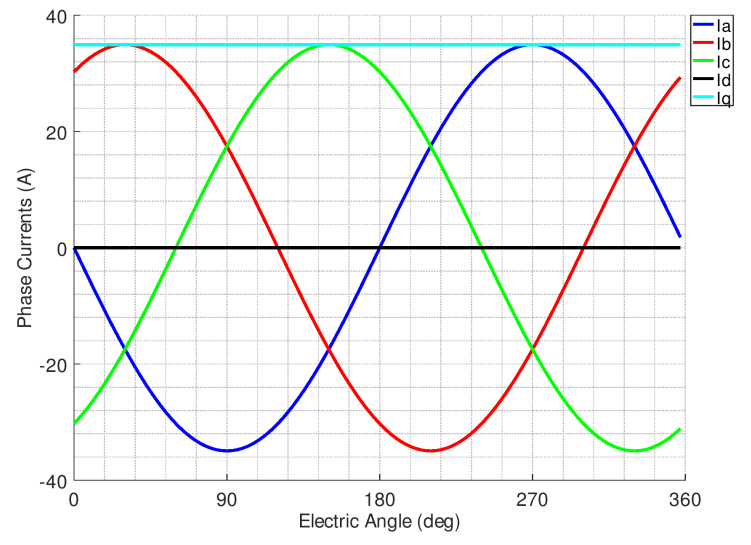

(a)

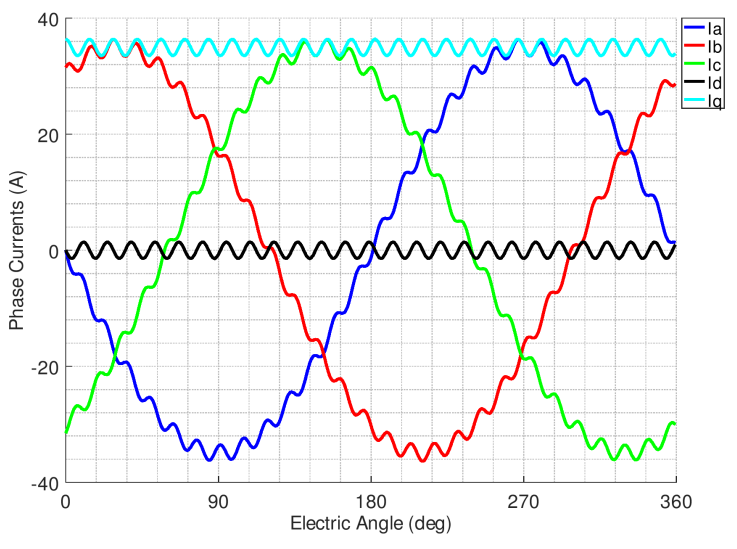

(b)

Figure 7. Finite element analysis (FEA) simulations of IPM at $\overrightarrow{j_{R M S}}=6.7 \mathrm{~A}_{\mathrm{RMS}} / \mathrm{mm}^{2}$ : (a) sinusoidal phase currents; (b) phase current with current ripple due to a PWM of $4 \mathrm{kHz}$.

The proposed IPM motor has the same stator lamination and stack length of the SPM machine and it was designed in order to achieve the torque of the reference SPM motor. Figure 8 compares the mean torques of the two motors when sinusoidal currents are imposed. Figure 9 compares the mean torques of the two motors when the current ripple is super imposed on the fundamental sinusoidal currents. 


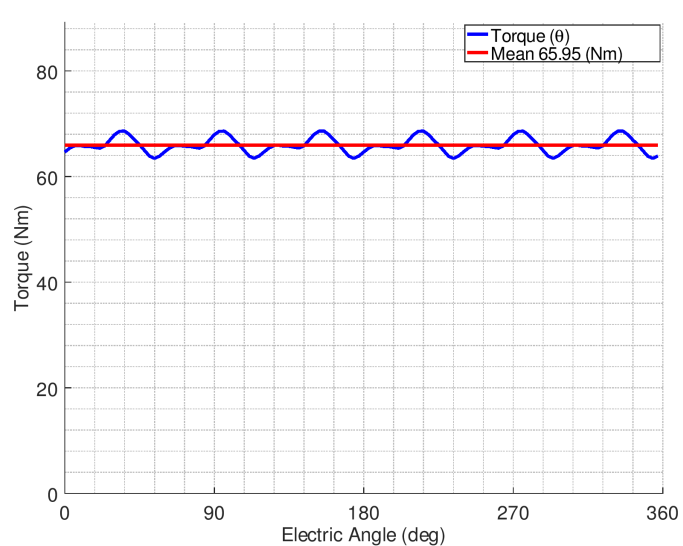

(a)

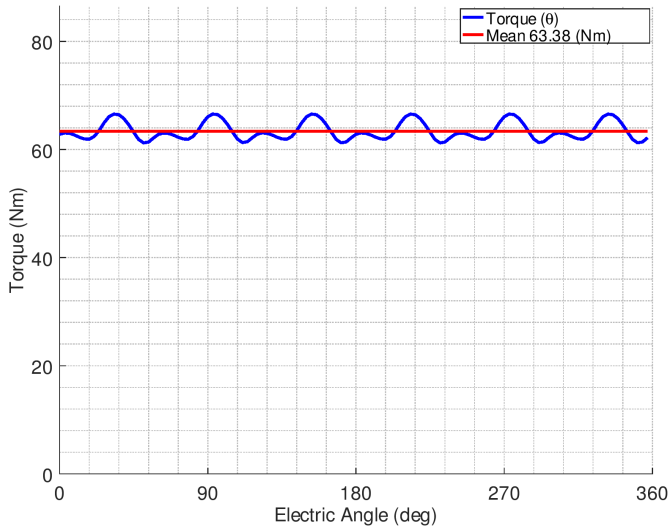

(b)

Figure 8. Torque profiles with sinusoidal currents: (a) reference SPM; (b) proposed IPM.

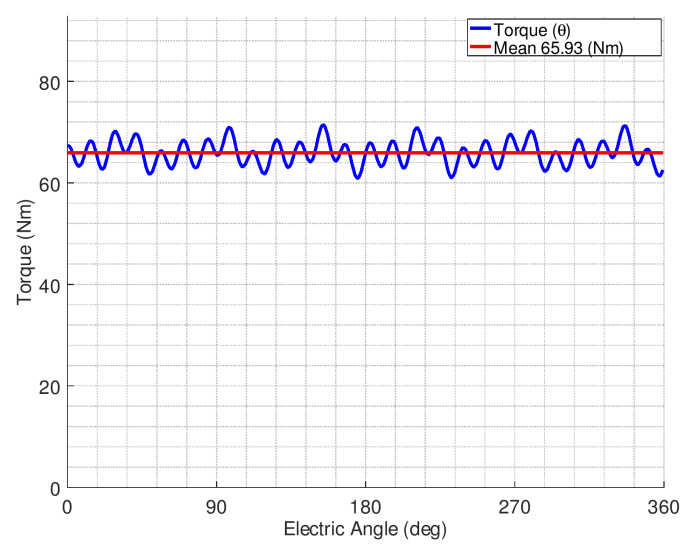

(a)

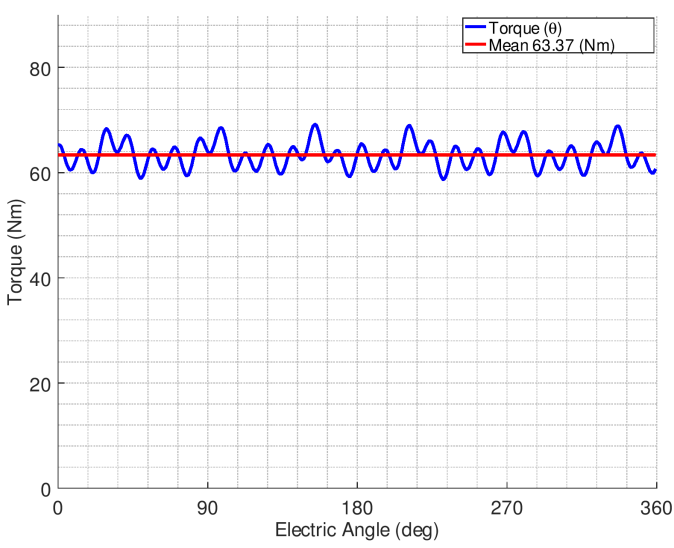

(b)

Figure 9. Torque profiles with current ripple of $4 \mathrm{kHz}$ : (a) reference SPM; (b) proposed IPM.

Figures 10 shows the power losses of the two synchronous motors at rated condition either with pure sinusoidal phase currents or with current ripple.

The FEA results demonstrated that the proposed IPM solution features lower power losses than the reference SPM in both supply conditions, see Table 2. At fixed mechanical speed of $3000 \mathrm{rpm}$, the current ripple induces an increase of motor total power losses of $52.8 \%$ for the reference SPM motor and $37 \%$ for the proposed IPM motor.

Table 2. Power losses of reference SPM and proposed IPM motors.

\begin{tabular}{ccccc}
\hline Power Loss Component & SPM No Ripple & IPM No Ripple & SPM with Ripple & IPM with Ripple \\
\hline Joule Losses (W) & 391.07 & 390.15 & 368.12 & 367.26 \\
PMs Losses (W) & 45.06 & 2.78 & 368.02 & 220.43 \\
Iron Losses (W) & 138.72 & 155.15 & 141.23 & 163.43 \\
\hline Stator Iron Losses (W) & 138.70 & 142.18 & 140.23 & 148.62 \\
Rotor Iron Losses (W) & 0.03 & 12.96 & 0.39 & 14.81 \\
\hline Total Losses (W) & 574.86 & 548.08 & 877.37 & 751.12 \\
\hline
\end{tabular}

As for magnet losses, the proposed IPM outperforms the reference SPM; with perfect sinusoidal currents the magnet losses in the IPM are $6.2 \%$ of the magnet losses in the SPM motor, while, at a switching frequency of $4 \mathrm{kHz}$, the losses in the IPM motor are $59.8 \%$ of the SPM motor. 
It is worth noticing that the proposed IPM motor features reduced power losses, even if iron losses are higher than those in the reference SPM motor.

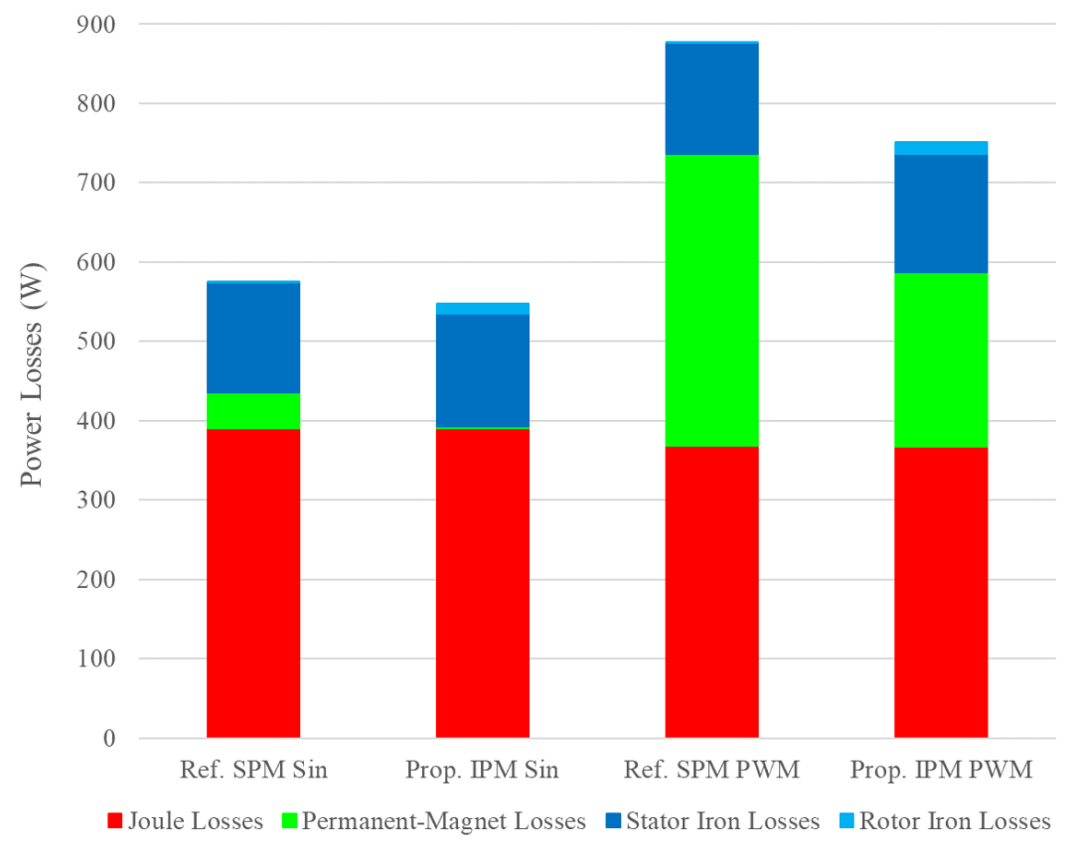

Figure 10. Comparison of power losses composition at $3000 \mathrm{rpm}$.

\subsection{Thermal Analysis of Reference and Proposed Machines by FEA}

A thermal simulation was performed to assess magnets temperature. The thermal simulations were carried out in static conditions imposing the losses evaluated in the magnetic simulation (see Table 2) as heat source for the copper, the lamination, and the magnets.

The internal magnets of the IPM motor are protected by the rotor lamination from the air gap magneto-motive force harmonics, so that heat exchange keeps the magnets at the same temperature of rotor laminations. This is confirmed by Figure 11a.

On the other hand, in the SPM machine, the magnets are exposed to the air gap harmonics and their external surface is cooled by the air in the air gap whose thermal conductivity is much smaller than the silicon-iron. Thus, the temperature of SPM magnets is much higher, Figure 11b. 


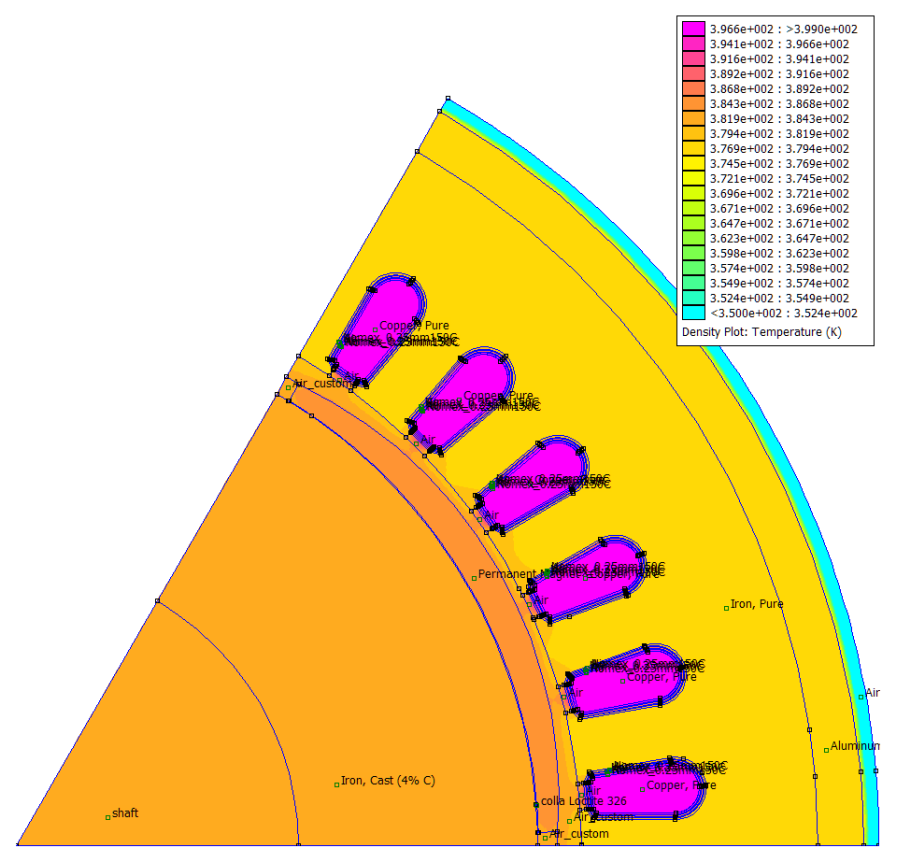

(a)

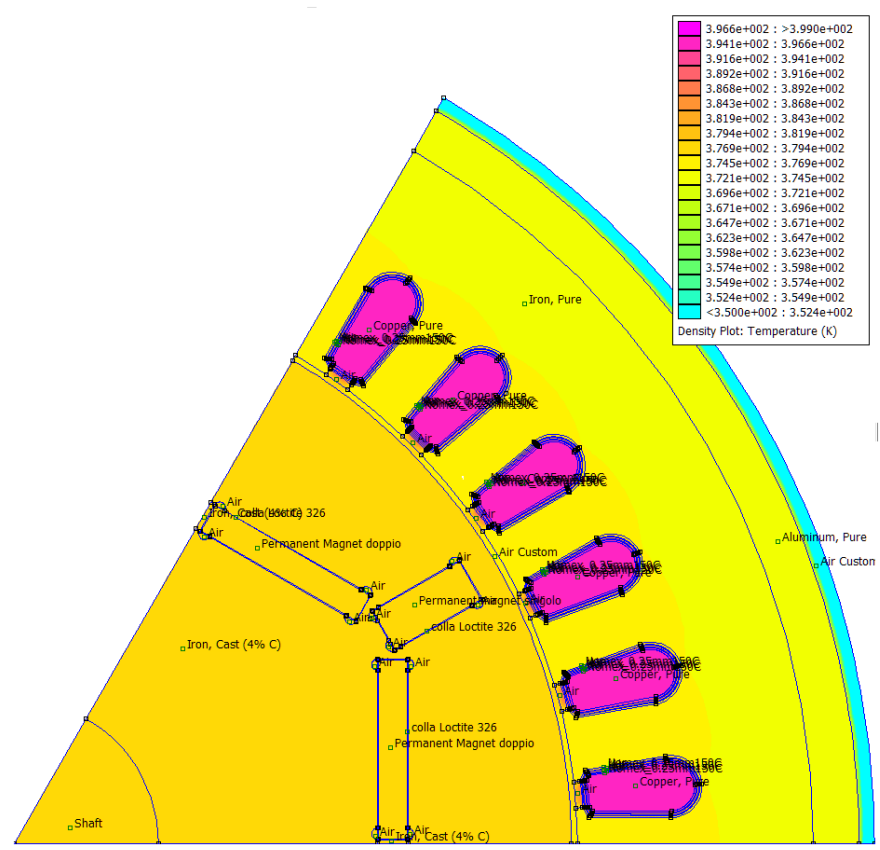

(b)

Figure 11. Steady-state thermal FEA with sinusoidal currents from $350 \mathrm{~K}$ to around $400 \mathrm{~K}$ : (a) reference SPM; (b) proposed IPM.

\section{Experimental Results}

Experimental tests were carried out on both SPM and IPM motors. The IPM rotor temperature can only be measured by a thermal camera, while for SPM the internal temperature was measured by both thermocouples and a thermal camera. A first set of tests was carried out only on SPM machine thanks to the thermocouples, aiming at defining the relationship between magnet temperature and switching frequency. A final set of tests was carried out on both motors, aiming at measuring the temperature of the magnets at rated conditions and thermal equilibrium. 


\subsection{Thermal Test on SPM Motor with Thermocouple}

This set of tests on SPM motor was carried out linking the drive and the motor with PWM filters that limit the impact of current ripple. This decouples the dependency of magnet temperatures from current ripple, producing a relationship between magnet temperature and switching frequency.

Stator and rotor temperatures were measured by a set of thermocouples of K-type. Stator windings temperature was measured placing four pairs of thermocouples in different locations of the stator winding, as shown in Figure 12a,b. A special thermocouple set-up was used to measure PMs temperature: four pairs of thermocouples were placed on PMs surface, as shown in Figure 12c,d. Subsequently, the thermocouples were located inside a custom hole in the rotor shaft and connected outside on the shaft side.

During the tests, once the stator windings reached the thermal equilibrium, the motor was stopped and the PMs temperature was sampled connecting rotor thermocouples to the measurement equipment. The temperature profiles of stator windings at $4 \mathrm{~Hz}$ and $8 \mathrm{KHz}$ are shown in Figures 13a and 14a, respectively. The periodic drops of temperature are in correspondence with motor stops.

Table 3 reports the average temperatures values measured during different test conditions. The thermal stress is inversely proportional to the switching frequency, and it is reduced with a higher number of input filter stages, as this results in a more sinusoidal current signal. Without input filters, the temperature of reference SPM motor was around $120^{\circ} \mathrm{C}$ with a switching frequency of $8 \mathrm{kHz}$, and at a similar value with a switching frequency of $4 \mathrm{kHz}$ and one filter stage.

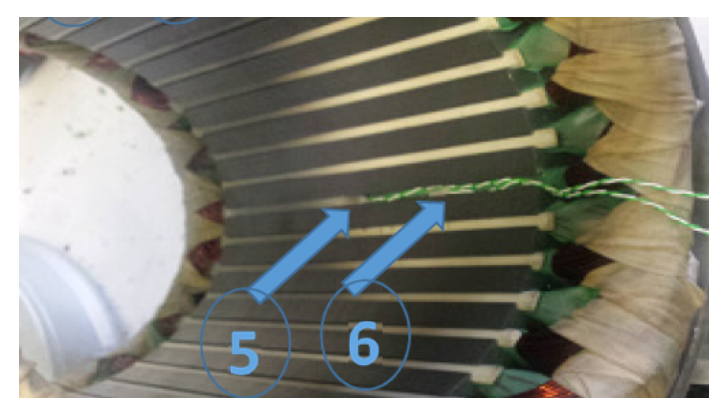

(a)

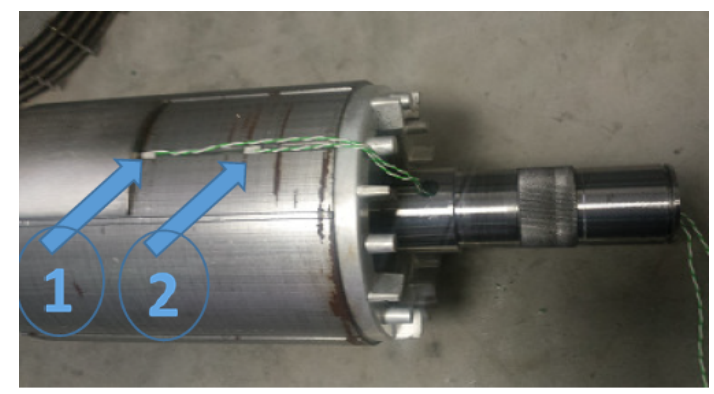

(c)

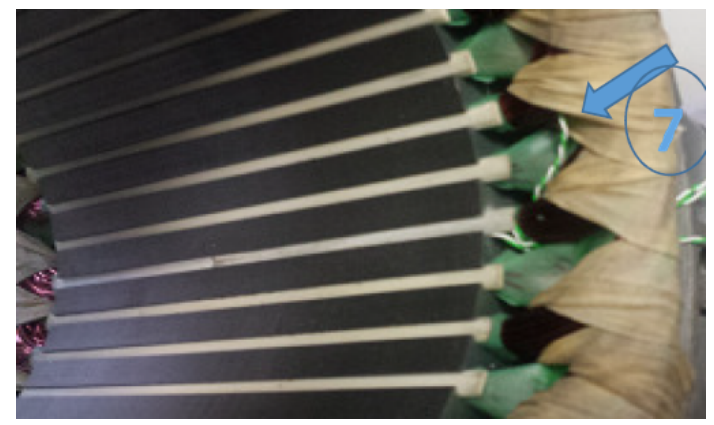

(b)

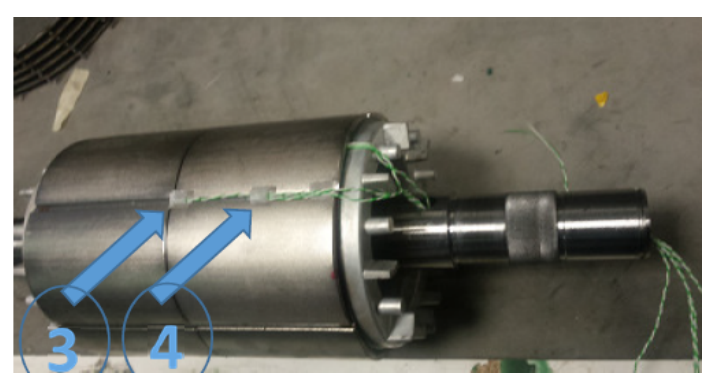

(d)

Figure 12. Thermocouples locations on SPM machine: $(\mathbf{a}, \mathbf{b})$ on stator winding; $(\mathbf{c}, \mathbf{d})$ on rotor permanent-magnets. 


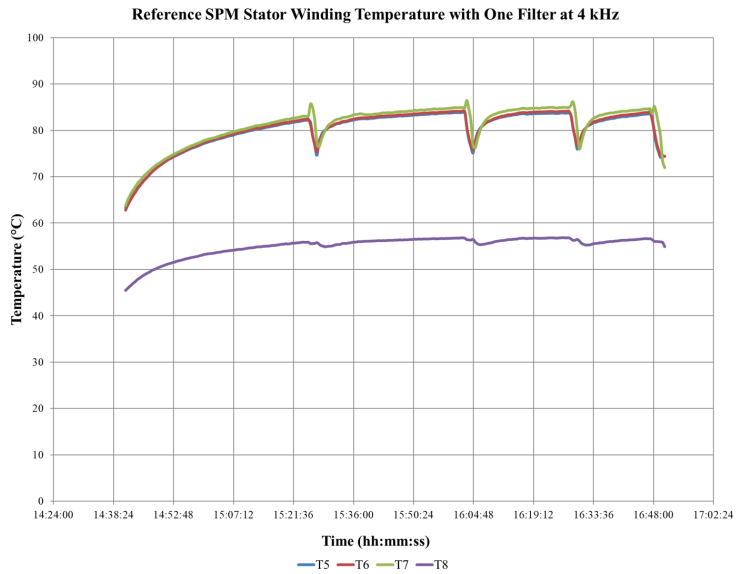

(a)

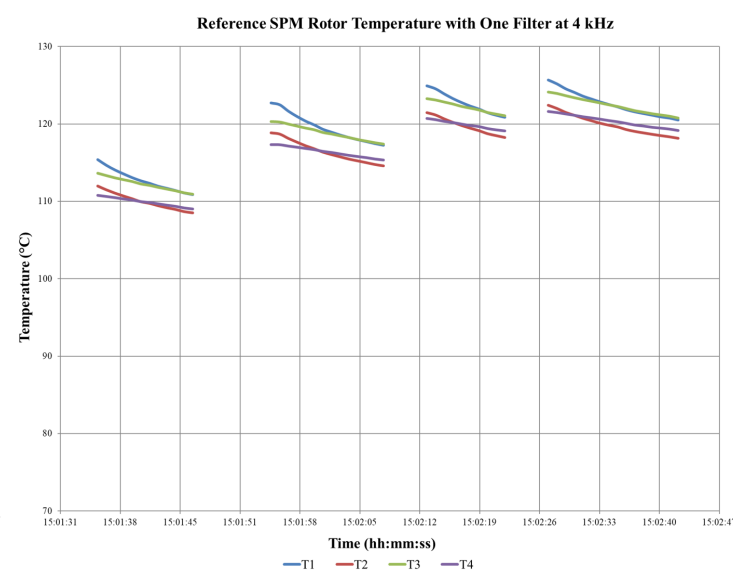

(b)

Figure 13. Temperature profiles during thermal equilibrium with one resistor-inductor (RL) filter at $4 \mathrm{kHz}$ with three, two and one input filters: (a) thermocouples on stator winding; (b) thermocouples on permanent-magnets .

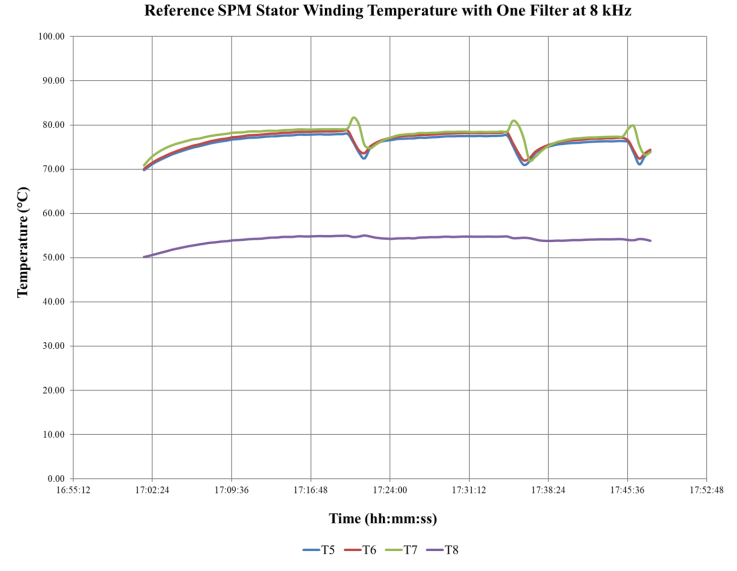

(a)

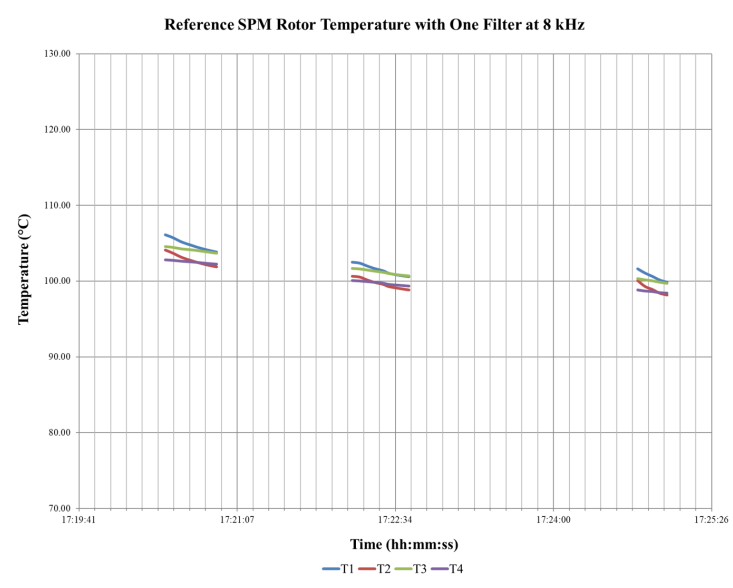

(b)

Figure 14. Temperature profiles during thermal equilibrium with one resistor-inductor (RL) filter at $8 \mathrm{kHz}$ with three, one and zero input filters: (a) thermocouples on stator winding; (b) thermocouples on permanent-magnets .

Table 3. Stator windings and permanent-magnet temperatures of the reference SPM motor at different PWM switching frequencies and input filtering conditions.

\begin{tabular}{ccccc}
\hline & \multicolumn{2}{c}{$\mathbf{4 ~ k H z}$} & \multicolumn{2}{c}{$\mathbf{8 ~ k H z}$} \\
\hline Description & Stator Temp. ${ }^{\circ} \mathbf{C}$ & Rotor Temp. ${ }^{\circ} \mathbf{C}$ & Stator Temp. ${ }^{\circ} \mathbf{C}$ & Rotor Temp. ${ }^{\circ} \mathbf{C}$ \\
\hline Without Input Filters & - & - & 88.80 & 119.58 \\
One Input Filter & 82.51 & 118.01 & 76.32 & 101.38 \\
Two Input Filters & 74.72 & 94.09 & - & - \\
Three Input Filters & 68.58 & 81.28 & 65.11 & 73.90 \\
\hline
\end{tabular}




\subsection{Thermal Tests on SPM and IPM with Thermal Camera}

In each test, both motors were operated at rated conditions with two different PWM switching frequencies: $4 \mathrm{kHz}$ and $8 \mathrm{kHz}$, without any PWM filter. The rotor temperatures were measured by the thermal camera with a special set-up of modified motor flange, Figure 15.

Figure 16a,b show the thermal photos of the reference SPM rotor at $8 \mathrm{kHz}$. As expected, the magnets temperature is close to $120^{\circ} \mathrm{C}$. The test at $4 \mathrm{kHz}$ has not been performed for SPM motor, because it causes demagnetization due to the magnet high working temperature.

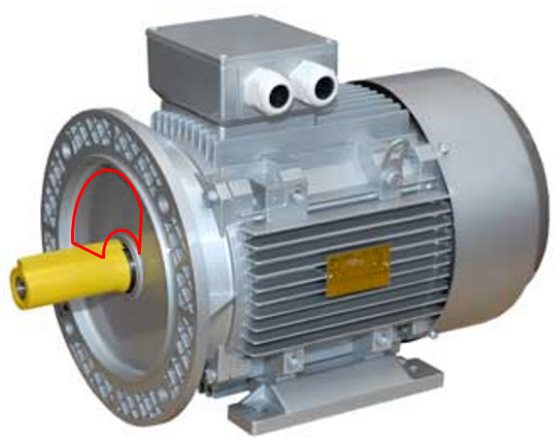

Figure 15. Position of the modified frontal motor flange.

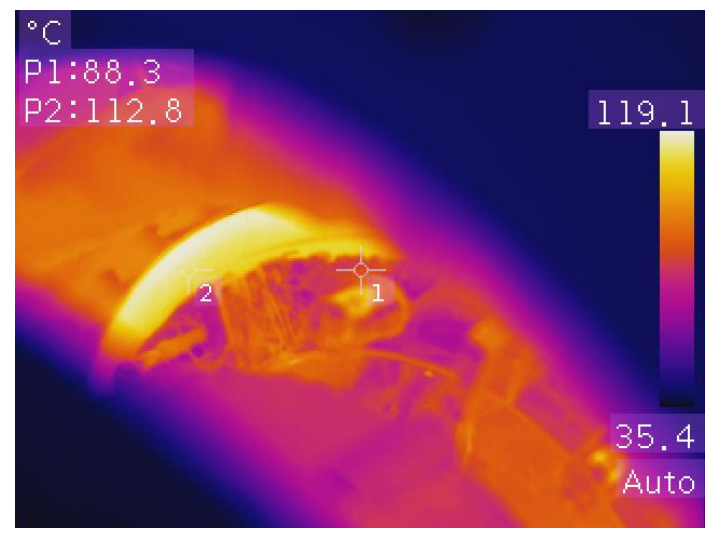

(a)

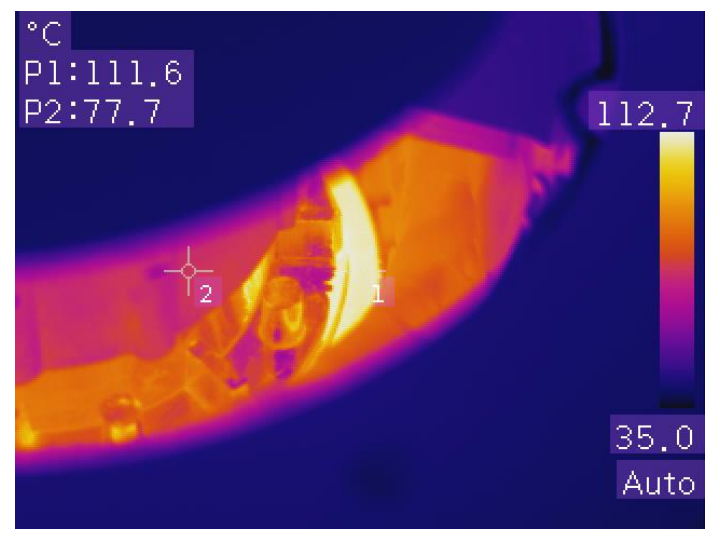

(b)

Figure 16. Thermal pictures acquired with thermal camera THT 70 (frame rate $50 \mathrm{~Hz}$ ) of reference SPM rotor taken with thermal camera: (a) thermocouples on stator winding; (b) thermocouples on permanent-magnets. The number of thermocouples is consistent with Figure 12.

The proposed IPM motor was also manufactured and tested at different PWM switching frequencies. Figure $17 \mathrm{a}, \mathrm{b}$ show the temperature of magnets for IPM at thermal equilibrium with a $4 \mathrm{kHz}$, i.e., at worst conditions from thermal point of view. The thermal camera shows that magnets and rotor are in the same temperature range, and that the magnets are far from demagnetization. 


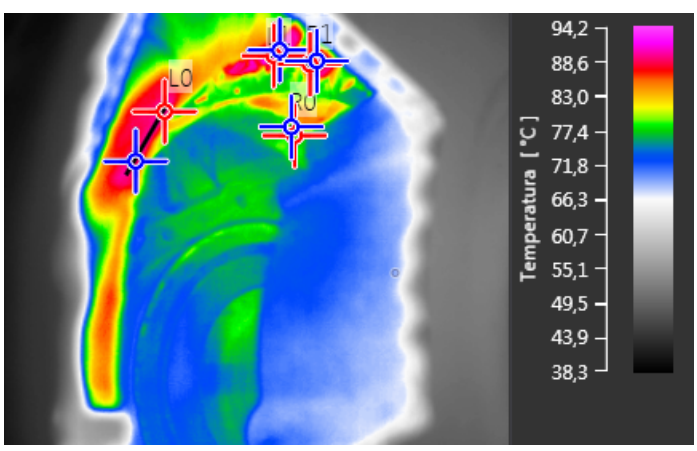

(a)

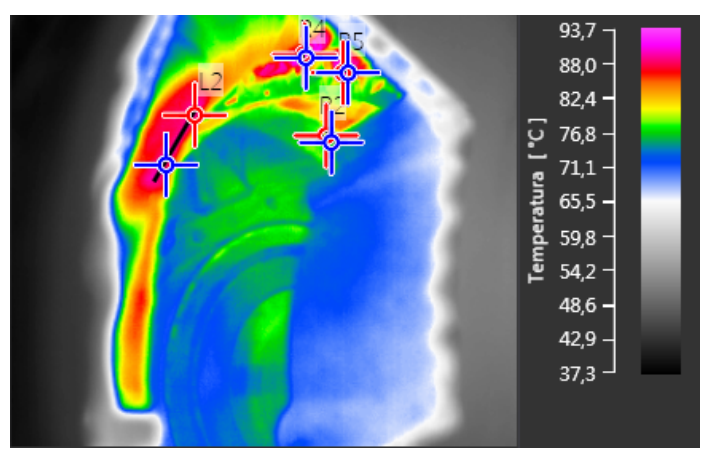

(b)

Figure 17. Thermal pictures acquired with thermal camera Workswell WIC 336 (frame rate $30 \mathrm{~Hz}$ ) of IPM-SM rotor at PWM frequency of $4 \mathrm{kHz}$ : (a) thermocouples on stator winding; (b) thermocouples on permanent-magnets. The number of thermocouples is consistent with Figure 12.

\section{Conclusions}

This paper shows an extensive analysis on permanent-magnet losses at different PWM switching frequencies. The aim was to improve the demagnetization fault-tolerant resistance of a low-cost surface permanent-magnet synchronous motor with distributed winding for industrial application.

The reference SPM motor was prone to PMs demagnetization, because the inverter PWM switching frequency superimposes additional time harmonics to stator phase currents, which induce eddy currents in conductive rotor parts, such as PMs. The additional eddy currents increases PMs working temperature because of Joule losses that could lead to serious and irremediable demagnetization.

The rotor geometry reference SPM motor was re-designed toward an IPM topology to protect PMs against eddy currents and, thus, demagnetization, keeping the same stator structure.

The performance of the reference SPM and proposed IPM motors was extensively analyzed and compared via magneto-static and thermal bi-dimensional FEA and experimental tests. Two motor prototypes were manufactured in order to assess machine fault-resistance against demagnetization.

Simulations and experiments show that the proposed IPM better protects against demagnetization, at any conditions.

The FEA results show a reduction of the total losses in both pure sinusoidal stator current and with current ripple. The estimated magnet losses in the IPM motor with a PWM frequency of $4 \mathrm{kHz}$ are the $59.8 \%$ of the SPM motor, this behavior has been confirmed by the reduction of the rotor and magnet temperature of more than $30^{\circ} \mathrm{C}$ in the experimental results.

Author Contributions: Conceptualization and validation, A.T. and M.D.; data curation, C.B.; methodology; and supervision and writing-review and editing, A.B. All authors have read and agreed to the published version of the manuscript.

Funding: This research received no external funding.

Conflicts of Interest: The authors declare no conflict of interest.

\section{Abbreviations}

The following abbreviations are used in this manuscript:

$\begin{array}{ll}\text { FEA } & \text { Finite Element Analysis } \\ \text { IEC } & \text { International Electrotechnical Commission } \\ \text { IM } & \text { Induction Motor } \\ \text { IPM-SM } & \text { Interior Permanent-Magnet Synchronous Motor } \\ \text { MMF } & \text { Magnetomotive Force } \\ \text { PM } & \text { Permanent-Magnet }\end{array}$


PWM Pulse Width Modulation

RMS Root Mean Square

SPM-SM Surface Permanent-Magnet Synchronous Motor

SPP Slots per Phase per Pole

VSI Voltage Source Inverter

\section{References}

1. International Electrotechnical Commission. Rotating Electrical Machines_Part 30-1: Efficiency Classes of Line Operated AC Motors (IE Code), IEC 60034-30-1, 1st ed.; 2014. Available online: https:/ / webstore.iec.ch (accessed on 8 July 2020).

2. Bianchini, C.; Davoli, M.; Pellegrino, G.; Immovilli, F.; Lorenzani, E. Low cost PM synchronous servo-applications employing asynchronous-motor frame. In Proceedings of the 2015 IEEE Energy Conversion Congress and Exposition (ECCE), Montreal, QC, Canada, 20-24 September 2015; pp. 6090-6095. doi:10.1109/ECCE.2015.7310513J.

3. Yamazaki, K.; Shina, M.; Kanou, Y.; Miwa, M.; Hagiwara, J. Effect of Eddy Current Loss Reduction by Segmentation of Magnets in Synchronous Motors: Difference Between Interior and Surface Types. IEEE Trans. Magn. 2009, 45, 4756-4759. doi:10.1109/TMAG.2009.2024159.

4. Yamazaki, K.; Kanou, Y.; Fukushima, Y.; Ohki, S.; Nezu, A.; Ikemi, T.; Mizokami, R. Reduction of magnet eddy current loss in interior permanent magnet motors with concentrated windings. In Proceedings of the 2009 IEEE Energy Conversion Congress and Exposition, San Jose, CA, USA, 20-24 September 2009; pp. 3963-3969. doi:10.1109/ECCE.2009.5316114.

5. Tessarolo, A. A survey of state-of-the-art methods to compute rotor eddy-current losses in synchronous permanent magnet machines. In Proceedings of the 2017 IEEE Workshop on Electrical Machines Design, Control and Diagnosis (WEMDCD), Nottingham, UK, 20-21 April 2017; pp. 12-19. doi:10.1109/WEMDCD.2017.7947717.

6. Yamazaki, K.; Fukushima, Y. Effect of Eddy-Current Loss Reduction by Magnet Segmentation in Synchronous Motors with Concentrated Windings. IEEE Trans. Ind. Appl. 2011, 47, 779-788. doi:10.1109/TIA.2010.2103915.

7. Yamazaki, K.; Abe, A. Loss Investigation of Interior Permanent-Magnet Motors Considering Carrier Harmonics and Magnet Eddy Currents. IEEE Trans. Ind. Appl. 2009, 45, 659-665. doi:10.1109/TIA.2009.2013550.

8. Huang, W.; Bettayeb, A.; Kaczmarek, R.; Vannier, J. Optimization of Magnet Segmentation for Reduction of Eddy-Current Losses in Permanent Magnet Synchronous Machine. IEEE Trans. Energy Convers. 2010, 25, 381-387. doi:10.1109/TEC.2009.2036250

9. Li, J.; Xu, Y.; Zou, J.; Wang, Q.; Liang, W. Analysis and Reduction of Magnet Loss by Deepening Magnets in Interior Permanent-Magnet Machines with a Pole/Slot Ratio of 2/3. IEEE Trans. Magn. 2015, 51, 8112004. doi:10.1109/TMAG.2015.2445787.

10. Boubaker, N.; Matt, D.; Enrici, P.; Nierlich, F.; Durand, G.; Orlandini, F.; Longère, X.; Ä̈gba, J.S. Study of eddy-current loss in the sleeves and Sm-Co magnets of a high-performance SMPM synchronous machine (10 kRPM, 60 kW). Electr. Power Syst. Res. 2017, 142, 20-28. doi:10.1016/j.epsr.2016.08.045.

11. Yamazaki, K.; Fukushima, Y.; Sato, M. Loss Analysis of Permanent-Magnet Motors with Concentrated Windings-Variation of Magnet Eddy-Current Loss Due to Stator and Rotor Shapes. IEEE Trans. Ind. Appl. 2009, 45, 1334-1342. doi:10.1109/TIA.2009.2023393.

(C) 2020 by the authors. Licensee MDPI, Basel, Switzerland. This article is an open access article distributed under the terms and conditions of the Creative Commons Attribution (CC BY) license (http:// creativecommons.org/licenses/by/4.0/). 